\title{
ARTICLE OPEN \\ Trimetazidine enhances myocardial angiogenesis in pressure overload-induced cardiac hypertrophy mice through directly activating Akt and promoting the binding of HSF1 to VEGF-A promoter
}

\author{
Hong-yang Shu ${ }^{1,2}$, Yi-zhong Peng ${ }^{3}$, Wei-jian Hang ${ }^{1,2}$, Min Zhang ${ }^{1,2}$, Lan Shen ${ }^{4}$, Dao-wen Wang ${ }^{1,2}$ and Ning Zhou ${ }^{1,2}$
}

Latest clinical research shows that trimetazidine therapy during the perioperative period relieves endothelial dysfunction in patients with unstable angina induced by percutaneous coronary intervention. In this study we investigated the effects of TMZ on myocardial angiogenesis in pressure overload-induced cardiac hypertrophy mice. Cardiac hypertrophy was induced in mice by transverse aortic constriction (TAC) surgery. TAC mice were administered trimetazidine ( $2.8 \mathrm{mg} / 100 \mu \mathrm{L}$, i.g.) for 28 consecutive days. We showed that trimetazidine administration significantly increased blood vessel density in the left ventricular myocardium and abrogated cardiac dysfunction in TAC mice. Co-administration of a specific HSF1 inhibitor KRIBB11 (1.25 mg/100 $\mu \mathrm{L}$, i.h.) abrogated the angiogenesis-promoting effects of trimetazidine in TAC mice. Using luciferase reporter and electrophoretic mobility shift assays we demonstrated that the transcription factor HSF1 bound to the promoter region of VEGF-A, and the transcriptional activity of HSF1 was enhanced upon trimetazidine treatment. In molecular docking analysis we found that trimetazidine directly bound to Akt via a hydrogen bond with Asp292 and a pi-pi bond with Trp80. In norepinephrine-treated HUVECs, we showed that trimetazidine significantly increased the phosphorylation of Akt and the synergistic nuclear translocation of Akt and HSF1, as well as the binding of Akt and HSF1 in the nucleus. These results suggest that trimetazidine enhances myocardial angiogenesis through a direct interaction with Akt and promotion of nuclear translocation of HSF1, and that trimetazidine may be used for the treatment of myocardial angiogenic disorders in hypertensive patients.

Keywords: cardiac angiogenesis; pressure overload-induced cardiac hypertrophy; HSF1; VEGF-A; Akt; trimetazidine

Acta Pharmacologica Sinica (2022) 43:2550-2561; https://doi.org/10.1038/s41401-022-00877-8

\section{INTRODUCTION}

It is estimated that about $29.2 \%$ of the global population will suffer from hypertension by 2025 . However, the control rate of hypertension is $<15 \%$ [1-3]. The huge hypertensive population has produced 64.3 million heart failure patients, whose 5 -year mortality rate exceeds $50 \%[4,5]$. Heart failure also imposes a huge economic burden, estimated at $\$ 108$ billion per annum [6]. Therefore, exploring the molecular mechanisms and drugs related to hypertension and heart failure has always been an important topic in clinical and scientific communities.

The rarefaction of capillary density causes myocardial hypoxia and contractile dysfunction. Angiogenesis dysfunction caused by pressure overload is a critical pathological process that deteriorates cardiac function [7]. Capillaries and myocytes grow in proportion to the increasing heart mass in physiological cardiac hypertrophy, and the disproportionate growth of capillaries and myocytes during pressure overload contributes to the formation of pathological cardiac hypertrophy and its transition to heart failure [8]. Thus, ameliorating angiogenesis dysfunction may provide a promising therapeutic strategy for the treatment of cardiac hypertrophy and heart failure.

Trimetazidine (TMZ) has been used in Europe for more than $\mathbf{4 0}$ years, and has been recommended as a second-line drug for stable angina pectoris by the European Society of Cardiology [9]. The accumulated evidence suggests that TMZ is not only effective for angina pectoris, but also for heart failure [10], peripheral vascular disease [11], contrast-induced kidney disease [12], and ischemiareperfusion injury [13]. TMZ was first discovered to improving energy metabolism by inhibiting long-chain 3-ketoacyl-CoA thiolytic enzymes in the mitochondria, thereby limiting the aerobic oxidation of fatty acids [14-16]. Numerous subsequent studies have revealed that $\mathrm{TMZ}$ also plays various other essential roles, such as inhibiting cardiomyocyte apoptosis, reducing the production of reactive oxygen species, and improving myocardial interstitial fibrosis and anti-inflammation [17-22]. The latest clinical research has demonstrated that $\mathrm{TMZ}$ therapy during the perioperative

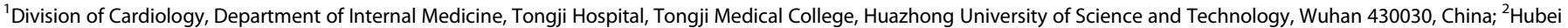

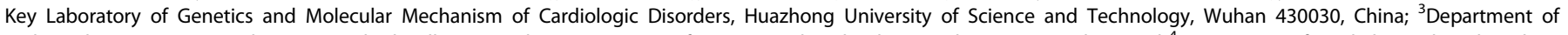

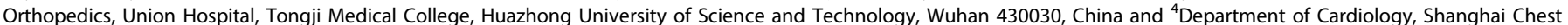
Hospital Shanghai Jiaotong University, Shanghai 200030, China

Correspondence: Ning Zhou (zhouning@tjh.tjmu.edu.cn)

Received: 13 September 2021 Accepted: 21 January 2022

Published online: 25 February 2022 
period relieves endothelial dysfunction in patients with unstable angina induced by percutaneous coronary intervention [23]. However, the underlying mechanism by which TMZ regulates myocardial angiogenesis has not yet been revealed. Therefore, Our work studied the regulatory effects of TMZ on angiogenesis and investigated the activation of Akt, nuclear translocation of HSF1 and sequential VEGF-A accumulation in in endothelial cells, aiming to clarify the underlying mechanism of TMZ in alleviating myocardial angiogenesis dysfunction and heart failure.

\section{MATERIALS AND METHODS}

Animal model and ethics approval

Male C57 mice (purchased from Beijing Charles River Laboratories) were used in this study. All animal experiments were conducted using procedures approved by the Institutional Animal Care in accordance with the NIH Guide for the Care and Use of Laboratory Animals. The protocol was approved by the Committee on the Ethics of Animal Experiments of the Animal Research Committee of Tongji College. Twenty-four mice included in the experiment were divided into the following four groups: sham, transverse aortic constriction (TAC), TAC + trimetazidine (TMZ (HY-B0968A, MedChemExpress, NJ, USA)), and TAC + TMZ + KRIBB11 (HY100872, MedChemExpress, NJ, USA) groups, with six mice in each group. After surgery, mice were administered either TMZ at 100 $\mu \mathrm{L} / 2.8 \mathrm{mg}$ or $100 \mu \mathrm{L}$ PBS by gavage. No mice died after these treatment. Four weeks after surgery, ultrasound and histological staining were used to detect changes in the heart.

\section{Transverse aortic constriction (TAC)}

TAC surgery was performed on 12-week-old male C57BL/6J mice as described previously [24]. Briefly, the mice were anesthetized and placed in a supine position on an operating table. After tracheal intubation, the mice were artificially ventilated at a tidal volume of $2-3 \mathrm{~mL} / \mathrm{h}$, and a respiratory rate of $90-110$ beats per minute (BPM) was maintained using a dedicated mouse ventilator. An incision was made in the chest wall at the second intercostal space. The mouse aorta was then hooked out with a homemade hook connected with a string through the incision, and the tissues surrounding the blood vessel were separated. Next, a 27G needle was placed between the aorta and ligature. After ligation, the needle was withdrawn, and the chest cavity was closed and sutured layer-by-layer. Cardiac ultrasound was performed 2 weeks after TAC to validate the success of the model.

\section{Ultrasound detection}

Mice were anesthetized with $2 \%$ isoflurane and their limbs were firmly fixed on the console with paper tape, and then the flow rate of $2 \%$ isoflurane was adjusted, while screening $\mathrm{HR}$, to control the HR between 550 and 600 BPM. The Visualsonics Vevo 1100 system (Vevo 1100, FUJIFILM VisualSonics, Japan) and a $30 \mathrm{~Hz}$ probe were used to detect the left ventricular long axis and short axis sections of the mice and collect relevant information including left ventricular internal diameter, left ventricular anterior wall thickness, left ventricular posterior wall thickness, and ejection fraction from at least three cardiac cycles.

\section{Cell culture}

HUVECs (Our laboratory preserved cell lines) were seeded onto 6-well plates containing RPMI-1640 medium (11875101, Thermofisher Scientific, MA, USA) supplemented with 10\% FBS (10091, Thermofisher Scientific, MA, USA) and then placed in a $37^{\circ} \mathrm{C}$ cell incubator. The medium was replaced with RPMI-1640 medium without FBS $24 \mathrm{~h}$ before intervention. After incubation with epinephrine (PE, $100 \mu \mathrm{M}, \mathrm{HY}-\mathrm{B} 0447 \mathrm{~B}$, MedChemExpress, NJ, USA), TMZ $(5 \mu \mathrm{M}), \mathrm{KRIBB} 11(10 \mu \mathrm{M})$, and AZD5363 (10 nM, HY15431, MedChemExpress, NJ, USA) for $24 \mathrm{~h}$, the cells were collected for subsequent experiments.
Molecular docking of Akt and TMZ

The structure of Akt (PDB:6S9W) (resolution: 2.30 ̊) was downloaded from the protein data bank (http://www.rcsb.org/), and the structure of TMZ was constructed using ChemSketch 12.0 (ACD Systems, Canada). These two structures were imported into the program Schrodinger Suites 2018 (Schrodinger, Inc. CA, USA). The Protein Preparation Wizard tool [25] was used to optimize the structure of Akt by removing water molecules and arranging bond orders. Likewise, the structure of TMZ was optimized using the Ligand Preparation tool to neutralize the structure and generate a possible tautomer. Additionally, conformation alteration was allowed in the docking calculation. Next, a ligand docking tool was used to calculate the docking score and determine the possible bonding site and interactions between the ligand and protein.

\section{Immunofluorescence staining}

The distribution of HSF1 was assessed by immunofluorescence as described previously [26]. Briefly, the cells were fixed with 4\% paraformaldehyde (G1101-500ML, Servicebio, Wuhan, China) for $15 \mathrm{~min}$, permeabilized with $1 \%$ Triton X-100 (G5060-100ML, Servicebio, Wuhan, China) for $10 \mathrm{~min}$, and incubated overnight at $4{ }^{\circ} \mathrm{C}$ with anti-HSF1 primary antibody (A13765, 1:100, Abclonal, Wuhan, China). Next, the cells were incubated with Cy3-labeled goat anti-rabbit antibody (BA1032, Boster, Wuhan, China) for $1 \mathrm{~h}$ and DAPI (AR1177, Boster, Wuhan, China) for $15 \mathrm{~min}$ at room temperature. The distribution of HSF1 was then observed by fluorescence microscopy (IX73-A12FL/PH, OLYMPUS, Japan).

\section{CCK8 assay}

HUVEC proliferation was detected using CCK8 assay as described previously [27]. Briefly, the cells to be tested were scraped from a 6-well plate to prepare a cell suspension and seeded onto a 96well plate at a density of 1000 cells per well. The 96 -well plate was incubated at $37^{\circ} \mathrm{C}$ for $4-6 \mathrm{~h}$, and after $1-4 \mathrm{~h}$ of incubation with 10 $\mu \mathrm{L}$ of CCK8 (CCK8, Dojindo Laboratories, Japan), the absorbance at $450 \mathrm{~nm}$ was measured.

\section{5-ethynyl-2'-deoxyuridine (EdU) staining}

The proliferation of HUVECs was detected with EdU staining as described previously [28]. Briefly, $2 \mathrm{~mL}$ of medium containing $10 \mu \mathrm{M}$ EdU (ab222421,abcam,UK) was added to each well of a 6-well plate. After incubating the cells for $2 \mathrm{~h}$ at $37^{\circ} \mathrm{C}$, the culture medium was discarded, 4\% paraformaldehyde fixative and 0.5\% Triton X-100 for permeation were added sequentially, and the cells were incubated in Apollo staining solution at $37^{\circ} \mathrm{C}$ for $30 \mathrm{~min}$ in the dark. Next, the cells were observed using a fluorescence microscope.

\section{Dual-luciferase reporter assay}

The promoter regions of wild-type and mutant VEGF-A genes were cloned into the pGL4 basic luciferase reporter vector, and HSF1 was cloned into the pcDNA3.1 (+) plasmid (V79020, Thermofisher Scientific, MA, USA). All vectors were verified by sequencing. Mouse endothelial cells were seeded onto a 24-well culture dish, and each well was transfected with wild-type or mutant VEGF-A promoter luciferase reporter gene $(0.5 \mu \mathrm{g})$ and HSF1 $(0.5 \mu \mathrm{g})$. Forty-eight hours after transfection, cell lysates were collected, and the Dual-Luciferase Reporter Assay kit (E2920, Promega, USA) was used to analyze the firefly and Renilla luciferase activity. The experiment was repeated three times. The ratio of firefly-to-Renilla luciferase activity was used as a standardization index for the luciferase activity of each group.

Electrophoretic mobility shift assay (EMSA)

The double-stranded VEGF-A DNA probe with or without the $5^{\prime}$ biotin label was used in EMSA. Equimolar complementary strands were mixed and gradually cooled to room temperature after incubating for $5 \mathrm{~min}$ at $95^{\circ} \mathrm{C}$. According to the manufacturer's 
instructions (GS009, Beyotime, Shanghai, China), the doublestranded DNA probe and nuclear extracts were thoroughly mixed in a $10-\mu \mathrm{L}$ reaction system. The complex was then separated on a $6 \%$ polyacrylamide gel containing $0.5 \times$ TBE and transferred to a positively charged nylon membrane. After UV crosslinking, the labeled DNA probes were detected with HRP-streptavidin.

\section{Chromatin immunoprecipitation (CHIP)-qPCR}

ChIP analysis was performed using the ChIP kit (P2078, Beyotime, Shanghai, China) according to the manufacturer's instructions as described previously [29]. Cells overexpressing HSF1 or the control vector were crosslinked with $1 \%$ formaldehyde at $37^{\circ} \mathrm{C}$ for $15 \mathrm{~min}$. The resulting cell pellet was then collected. The nucleus extracts were obtained with the nuclear protein and cytoplasmic protein preparation kit (P1200, Applygen, Beijing, China), and the chromatin was cut into a length of $\sim 200-400$ bp with ultrasound. The sheared nuclear extracts were incubated with anti-HSF1 antibody (sc-17757X, Santa Cruz, CA, USA) and protein A/G agarose beads (20422, Thermofisher Scientific, MA, USA) overnight at $4{ }^{\circ} \mathrm{C}$. Next, the protein A/G agarose beads-antibody-protein complex was collected and separated with elution buffer after decrosslinking. DNA was extracted with a DNA purification kit (DP304, TIANGEN, Beijing, China), and the precipitated DNA fragments were analyzed by real-time quantitative $P C R$. Primers of VEGF-A (sense: 5'-GAAGATGTGGAGAGTTGGAGGAA-3'; antisense: $5^{\prime}$-CCTGCGTGATGATTCAAACC-3'). The nuclear extracts were used as a positive control, and the nuclear extracts incubated with anti-lgG antibody (ab205718, abcam, UK) were used as a negative control. Each assay was repeated for three times.

Scratch wound healing assay

Scratch wound healing assay was performed as described previously [30]. $1 \times 10^{6}$ cells/well were seeded into a 6-well plate that has been coated with extracellular matrix (ECM) substrates (50 $\mathrm{\mu g} / \mathrm{mL}$ poly-L-lysine, C0313-5mg, Beyotime, Shanghai, China). The monolayer was scratched in a straight line by a pipette tip. Detached cells were then removed. Then remaining cells were cultured in a fresh medium supplemented with PE, TMZ, and HSF1 inhibitor for $12 \mathrm{~h}$. Mitomycin C $(10 \mu \mathrm{g} / \mathrm{mL}, \mathrm{HY}-13316$, MedChemExpress, NJ, USA), was also added to inhibit DNA replication. After the incubation, cell monolayers were fixed with $3.7 \%$ paraformaldehyde for $15 \mathrm{~min}$ and photographed at $0 \mathrm{~h}, 12 \mathrm{~h}$ post-wounding. Wound area was then calculated by manually tracing the cell-free area in images using ImageJ software and by the following formula: wound closure $\%=\left(A_{\mathrm{t}=0 \mathrm{~h}}-A_{\mathrm{t}=12 \mathrm{~h}}\right) / A_{\mathrm{t}=0 \mathrm{~h}} \times 100$, where $A_{\mathrm{t}=0 \mathrm{~h}}$ is the initial area (time zero), and $A_{\mathrm{t}=12 \mathrm{~h}}$ is the area of the wound measured $12 \mathrm{~h}$ post scratch.

Tube formation assay

Tubule formation assay was conducted as previously described [31]. Briefly, before seeding $2 \times 10^{4}$ cells/well, the pipet and 96well plates were precooled and coated with $50 \mu \mathrm{L}$ of Matrigel (356234, BD Biosciences, Bedford, MA, USA) for $30 \mathrm{~min}$ at $37^{\circ} \mathrm{C}$. After incubation for $6 \mathrm{~h}$ in $100 \mu \mathrm{L}$ endothelial culture medium containing different chemicals (PE $100 \mu \mathrm{M}$, AZD5363 $10 \mathrm{nM}$, KRIBB11 $10 \mu \mathrm{M}$ ) in incubator, HUVECs were captured by a bright filed microscope. Total tube length was analyzed to quantify the tube formation ability.

\section{Western blot}

HUVECs were lysed on ice for $30 \mathrm{~min}$ with the cell lysate. The protein concentration was measured using the BCA method (P0011, Beyotime, Shanghai, China) before being subjected to electrophoresis and transferred to a PVDF membrane (IPVH00010, Millipore, Germany). The PVDF membrane was incubated with antibodies against p-HSF1, HSF1, VEGF, p-Akt, Akt, and GAPDH overnight at $4{ }^{\circ} \mathrm{C}$ and finally exposed and photographed. The antibody information was listed in Supplementary Table S1.
Co-immunoprecipitation (Co-IP)

Co-IP experiments were performed as described previously [32]. Cells that had been transfected with the indicated plasmids were lysed with IP lysis buffer (P0013J, Beyotime, Shanghai, China). Before performing SDS-PAGE, the pre-cleared cell lysate was incubated with an appropriate amount of primary antibody at $4{ }^{\circ} \mathrm{C}$ overnight. Protein A/G agarose beads (sc-2003, Santa Cruz, CA, USA) were then used to precipitate the target protein. After performing SDS-PAGE, the proteins were immediately transferred to the PVDF membrane and incubated with antibodies against HSF1, Akt, and p-Akt. The protein of interest was then visualized by chemiluminescence using the Tanon system (Tanon 5200, Shanghai, China). The antibody information was listed in Supplementary Table S1.

\section{Statistical analysis}

All data are expressed as the mean \pm SEM. The statistic's normality was tested by D'Agostino and Pearson omnibus normality test. Comparison between two groups was analyzed by Student's $t$ test. Multiple group comparisons were analyzed by one-way or two-way ANOVA, followed by Tukey's multiple comparison test using GraphPad Prism 8.0 software. Mann-Whitney test and Kruskal-Wallis test would be applied when the data did not show a normal distribution. $P<0.05$, represents statistically significant values.

\section{RESULTS}

TMZ alleviated angiogenesis dysfunction and improved heart function in pressure overload-induced cardiac hypertrophy mice Mice (12 weeks old) were administered TMZ by gavage for 28 consecutive days following TAC surgery to investigate the role of TMZ in promoting angiogenesis. The ultrasound (Supplementary Fig. S1a-c), cardiac morphology (Supplementary Fig. S1a), and heart weight/tibia length (HW/TL) (Supplementary Fig. S1d) results demonstrated that TMZ effectively relieved myocardial hypertrophy under pressure overload, which manifested with increased heart weight, enlarged heart chambers, and increased thickness of the anterior and posterior walls of the left ventricular myocardium. The levels of cardiac hypertrophy markers, atrial natriuretic peptide, and B-type natriuretic peptide, also decreased with TMZ treatment (Supplementary Fig. S1f-h).

Immunofluorescence staining for CD31 was performed to detect the extent of blood vessel formation. As shown in Fig. 1a, the blood vessels decreased significantly in the TAC group compared with the sham group, whereas abundant blood vessels appeared in the TMZ treatment group. The quantification of angiogenesis, analyzed by the number of cells positively stained for CD31, verified the angiogenesis-promoting effects of TMZ (Fig. 1b). Western blotting also showed increased expression of CD31 and VEGF in mouse hearts treated with TMZ (Fig. 1d, e). The angiogenesis-stimulating function of $T M Z$ in vitro was confirmed using proliferation assays. As expected, fewer $\mathrm{EdU}^{+}$cells were observed in the PE group. However, TMZ accelerated cell proliferation, as shown by the much higher number of $\mathrm{EdU}^{+}$cells (Fig. 1f, g). Our data suggest that TMZ augmented the angiogenic responses and improved the heart function of pressure overloadinduced cardiac hypertrophy mice.

Inhibition of HSF1 activation restrained the angiogenic effect of TMZ

In our previous study, HSF1 was demonstrated to be critically involved in cardiac angiogenesis [33,34]. We then asked whether HSF1 was responsible for the angiogenesis-stimulating effect of TMZ. As detected by fluorescence staining and Western blot, fewer blood vessels and lower VEGF and CD31 expression were observed in the presence of HSF1 inhibitors (Fig. 2a-e). Correspondingly, to assess the effects of HSF1 on endothelial cells, a scratch wound assay was applied to measure cell migration ability. 
a
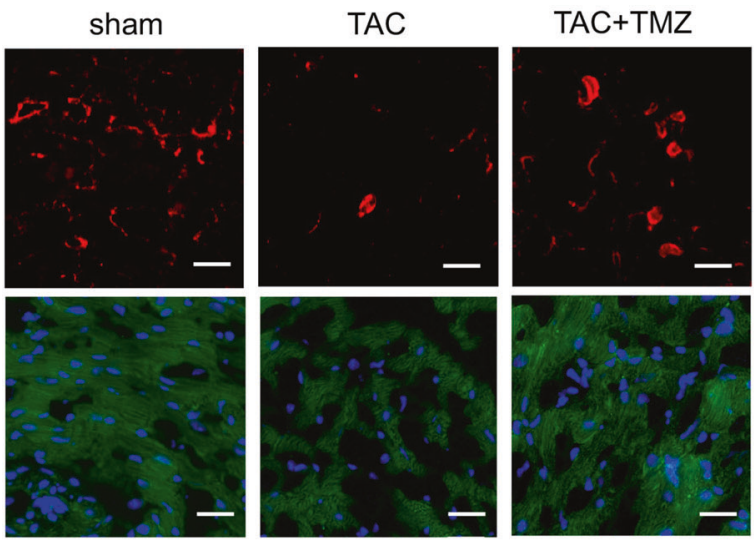

d

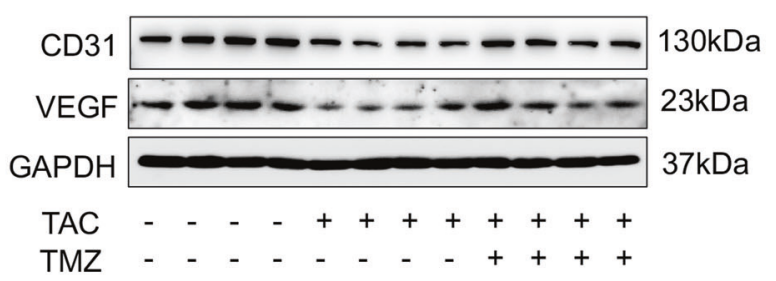

f
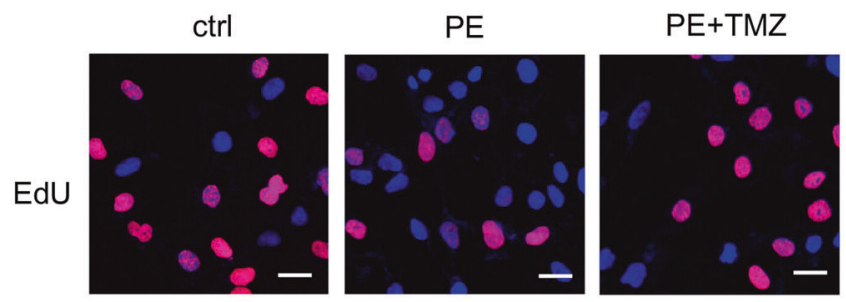

b

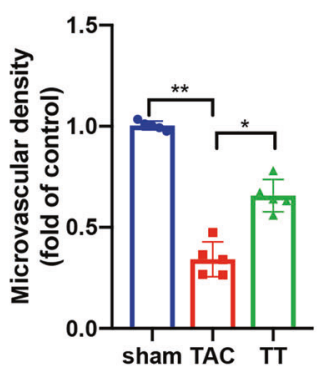

C

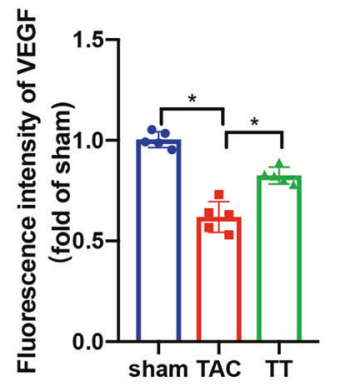

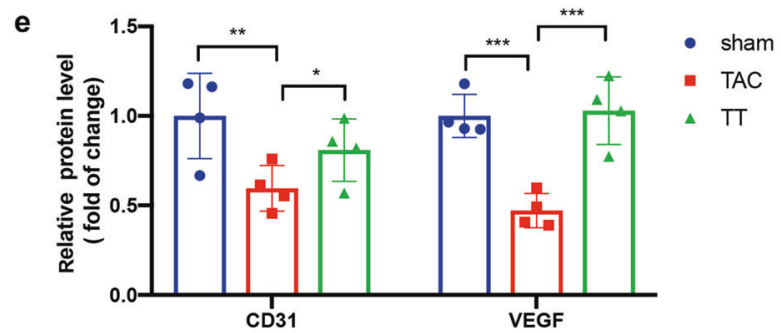

g

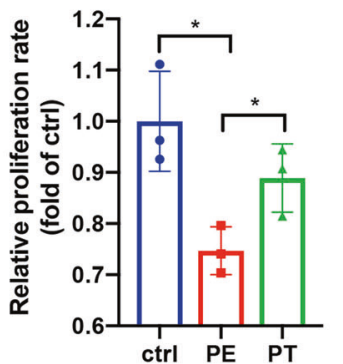

h

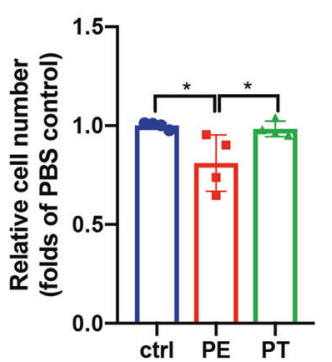

Fig. 1 TMZ alleviated angiogenesis dysfunction in pressure overload-induced cardiac hypertrophy mice. a Immunohistochemical staining of CD31 and VEGF was presented from left ventricular myocardium. Scale bar $=50 \mu \mathrm{m}$. b, c Quantification analysis of (a), $n=5$. d Western blotting of CD31 and VEGF, GAPDH was used as a loading control, $n=3$. e Quantification analysis of (d). f Representative EdU staining images of HUVECs. Scale bar $=20 \mu \mathrm{m} . \mathbf{g}$ Quantification analysis of (f). $\mathbf{h}$ CCK8 assay of HUVECs in indicated groups. Sham sham operation group, TAC TAC group, TT TAC + TMZ group, mice subjected to TAC surgery and treated with TMZ, TTHi TAC + TMZ + HSFi group, TAC mice were treated with HSF1 inhibitor (KRIBB11) and TMZ, Ctrl, PBS group, PE Norepinephrine group, PT PE + TMZ group, cells treated with PE and then treated with TMZ, PTHi PE + TMZ + HSFi group, cells were treated with HSF1 inhibitor (KRIBB11), TMZ and PE. Data were analyzed by two-way ANOVA analysis using GraphPad Prism 8 software $\left({ }^{*} P<0.05,{ }^{* *} P<0.01 ;{ }^{* *} P<0.01 ;{ }^{* * *} P<0.0001\right.$ vs. indicated group).

The results show that HSF1 inhibitor treatment markedly weakened the motility of HUEVCs, as determined by the larger wound closure area (Fig. 2f, g). The proliferation of HUEVCs was quantified by CCK8 analysis, EdU staining, and tube formation assay (Supplementary Fig. S3a, b). The results revealed that TMZ treatment caused a marked increase in proliferation. However, the effect was suppressed by the HSF1 inhibitor (Fig. 2h-j, Supplementary Fig. S2a, b). Collectively, these data suggest that TMZ enhances cardiac angiogenesis by HSF1.

TMZ promoted the binding of HSF1 with the VEGF-A promoter region

To further investigate the relationship between HSF1 and angiogenesis, we predicted several possible binding molecules for HSF1 from the JASPAR website, and found a specific binding site for HSF1 in the promoter region of VEGF-A. Consequently, we determined whether HSF1 bound to the VEGF-A promoter region, and if TMZ could enhance this binding. Dual-luciferase reporter assay was then carried out. The results revealed that HSF1 did combine with the promoter region of VEGF-A. TMZ strengthened this interaction, whereas the effect was reduced after HSF1 activity was inhibited (Fig. 3a). These data suggest that TMZ promoted the binding of HSF1 with the VEGF-A promoter region.

Four fragments of the VEGF-A promoter region $(-2000 /+37$, $-1300 /+37$ mutant, $-1300 /+37 \mathrm{WT}$, and $-500 /+37)$ were then cloned into a pGL3 fluorescent reporter gene vector to validate the HSF1-VEGF-A binding site. HSF1 significantly increased the fluorescence intensity of the $-2000 /+37 \mathrm{bp}$ and $-1300 /+37 \mathrm{bp}$ regions, while the other two regions showed no changes, indicating that the HSF1-VEGF-A promoter region binding site is located in the $1300 \mathrm{bp} / 500 \mathrm{bp}$ region; more accurately, the position is $-1192 \mathrm{bp} /-1178 \mathrm{bp}$ (Fig. 3b). The regulatory effects of the $-1192 \mathrm{bp} /-1178 \mathrm{bp}$ region were further evidenced by EMSA assay and CHIP-qPCR assay (Fig. 3c, d). 
a

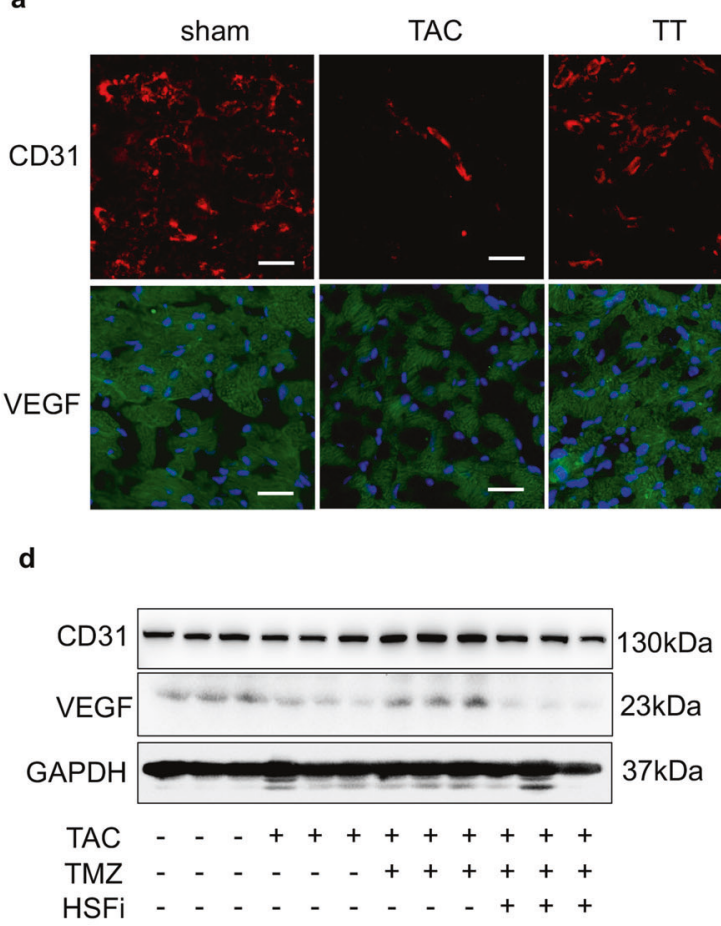

f
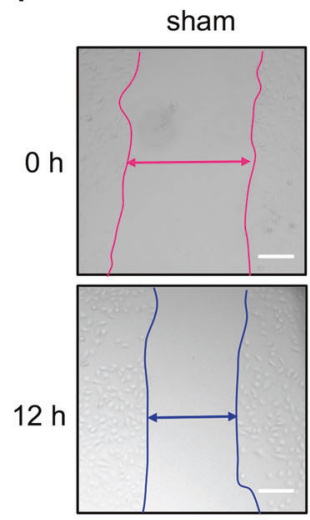

sham

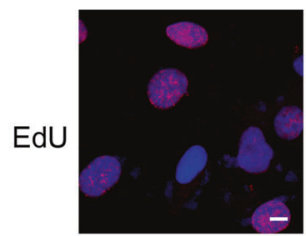

PE
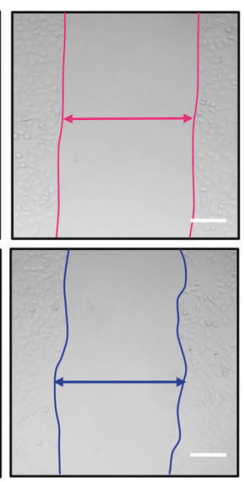

PE

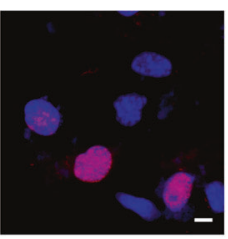

PT

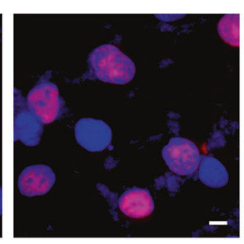

TTHi
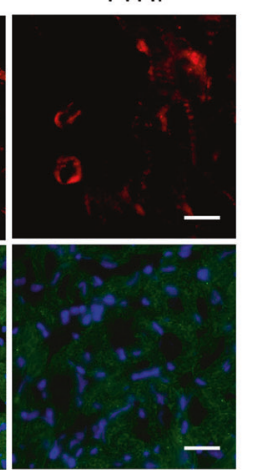

b

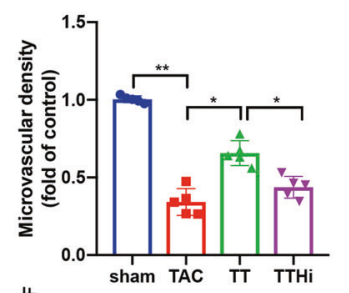

c

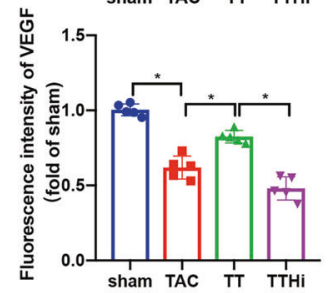

e

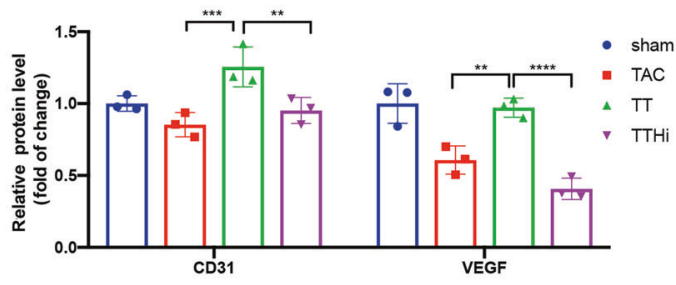

g
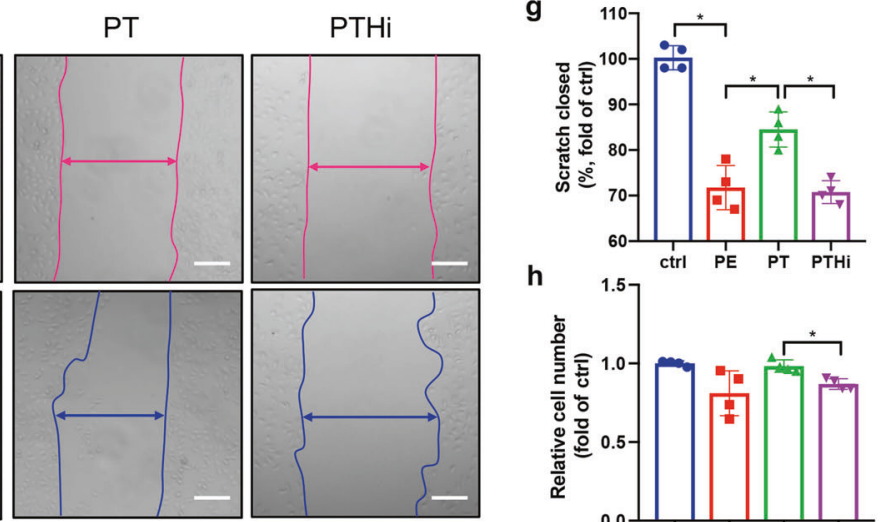

h

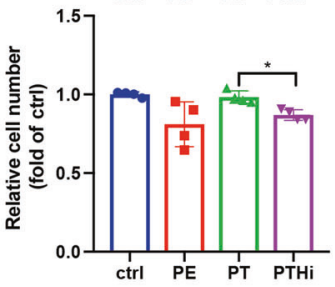

PTHi
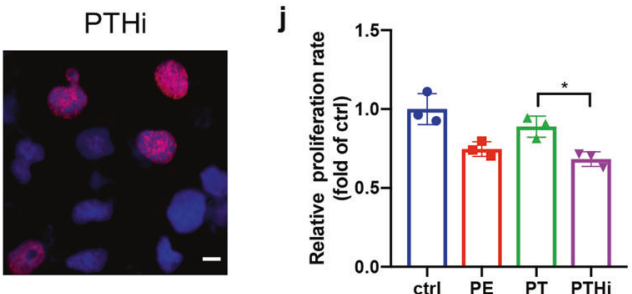

Fig. 2 HSF1 played a critical role in TMZ-mediated cardiac angiogenesis improvement. a Immunohistochemical staining of CD31 and VEGF was presented from left ventricular myocardium. Scale bar $=50 \mu \mathrm{m}$. b, c Quantification analysis of (a), $n=5$. d Western blotting of CD31 and VEGF, GAPDH was used as a loading control, $n=3$. e Quantification analysis of (d). f Representative migration images of HUVECs in $12 \mathrm{~h}$. Scale bar $=200 \mu \mathrm{m}$. g Relative scratch closed area of (f). $\mathbf{h}$ CCK8 assay of HUVECs in indicated groups. $\mathbf{i}$ Representative EdU staining images of HUVECs. Scale bar $=20 \mu \mathrm{m}$. j Quantification analysis of (i). Sham sham operation group, TAC TAC group, TT TAC + TMZ group, mice subjected to TAC surgery and treated with TMZ, TTHi TAC + TMZ + HSFi group, TAC mice were treated with HSF1 inhibitor (KRIBB11) and TMZ, Ctrl PBS group, PE Norepinephrine group, PT PE + TMZ group, cells treated with PE and then treated with TMZ, PTHi PE + TMZ + HSFi group, cells were treated with HSF1 inhibitor (KRIBB11), TMZ and PE, Data were analyzed by two-way ANOVA analysis using GraphPad Prism 8 software $\left({ }^{*} P<0.05,{ }^{* *} P<0.01 ;{ }^{* *} P<0.01 ;{ }^{* * *} P<0.0001\right.$ vs. indicated group). 


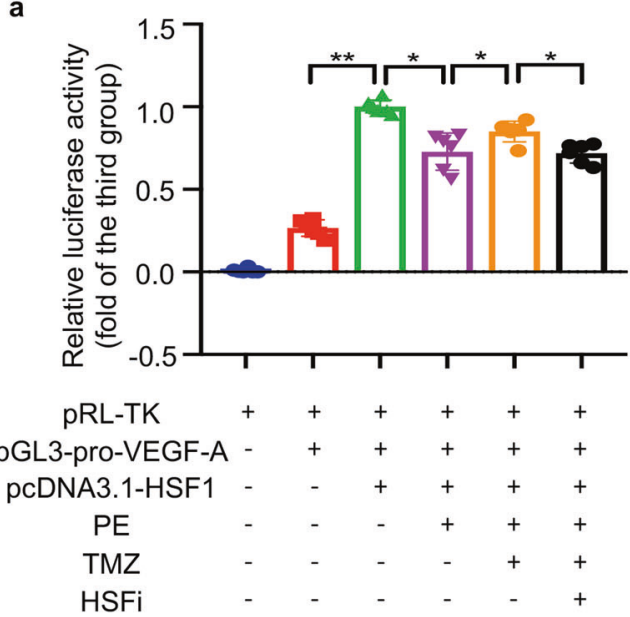

b

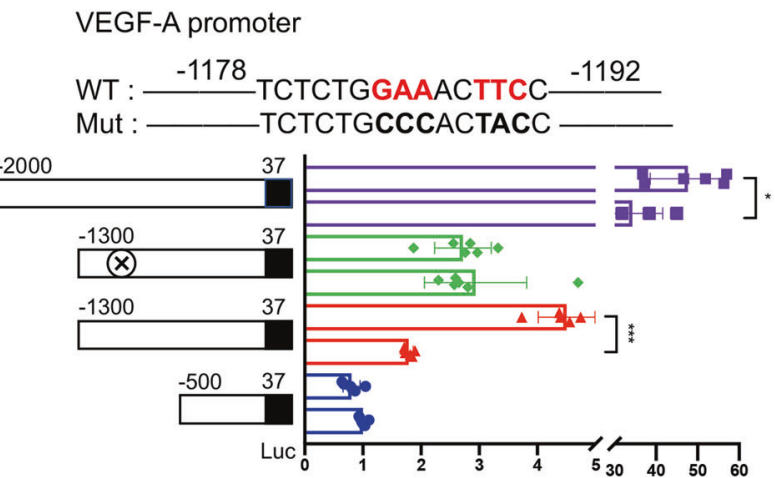

c VEGF-A promoter
WT : $\stackrel{-1178}{-}$ TCTCTGGAAACTTCC- -1192

Nuclear extract Labled $-1178 /-1192$ WT Unlabled -1178/-1192 WT Anti-HSF1

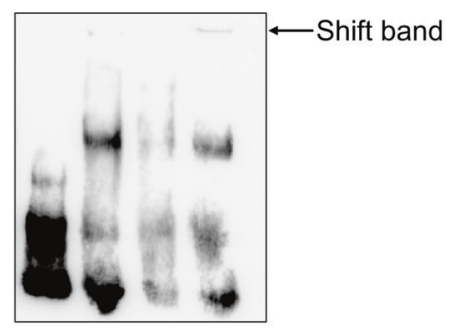

d

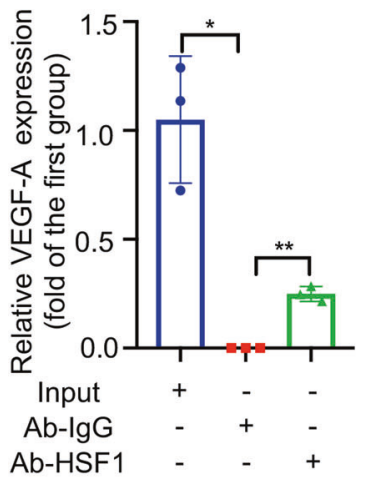

Fig. 3 HSF1 bound with VEGF-A promoter region. a Luciferase activity of pGL3-f reporter plasmids in indicated groups. b Luciferase activity of pGL3 $(-2000 /+37)$, pGL3 $(-1300 /+37)$, mutation pGL3 $(-1300 /+37)$, and pGL3 $(-500 /+37)$ reporter plasmids stimulated by pcDNA3.1HSF1, mutation region located in -1192 to -1178 . c Representative EMSA images with biotin-labeled DNA oligonucleotides VEGF-A-1192/ -1178-WT. d Verification of the interaction of HSF1 and the promoter region of VEGF-A by ChIP-qPCR. Ctrl PBS group, PE Norepinephrine group, PT PE + TMZ group, cells treated with PE and then treated with TMZ, PTHi PE + TMZ + HSFi group, cells were treated with HSF1 inhibitor (KRIBB11), TMZ and PE. Data were analyzed by two-way ANOVA analysis using GraphPad Prism 8 software ${ }^{*} P<0.5 ;{ }^{* *} P<0.01$, vs. indicated group).

TMZ activated HSF1 and promoted its nuclear translocation via Akt

We previously identified that TMZ activated Akt in a dosedependent manner from 1 to $5 \mu \mathrm{M}$ (Supplementary Fig. S2). To investigate whether HSF1 and Akt respond to TMZ treatment under pressure overload, PE-treated HUVECs were extracted, and Western blot was then used to detect changes in the phosphorylation level of HSF1 and Akt. As expected, HSF1S303 expression was elevated under PE treatment and reduced upon TMZ stimulation. In contrast, AktT473 expression decreased in response to PE treatment, while it increased in the presence of TMZ. These results indicate that HSF1 and Akt synchronously respond to TMZ (Fig. $4 a-c)$.

Specific inhibitors of Akt (AZD5363) and HSF1 (KRIBB11) were then used in subsequent experiments to further clarify the specific regulatory relationship between HSF1 and Akt. As shown in Fig. $4 d-i, T M Z$ could not activate HSF1 and promote its nuclear translocation following Akt inhibition, whereas it could still activate Akt when HSF1 was inhibited. These opposite results indicate that Akt is upstream of HSF1.

Next, HUVEC nucleoproteins and cytoplasmic proteins were separated to detect changes in the nuclear translocation of HSF1. Under TMZ treatment, the nuclear signals of HSF1 and p-Akt were significantly enhanced. However, they faded with the addition of the Akt inhibitor (Fig. 5a, b). Immunofluorescence analysis of HSF1 and p-Akt also showed that PE inhibited the phosphorylation of Akt, as well as the nuclear translocation of HSF1, while TMZ reversed these effects. The overlapping yellow parts in the nucleus suggest that HSF1 and Akt have a mutual binding relationship (Fig. 5c, d). Their binding relationship was also verified by Co-IP assay. As shown in Fig. 5e, f, HSF1 and Akt bound to each other without stimulation. The binding of Akt/p-Akt and HSF1 in the nucleus was significantly enhanced after TMZ treatment, and remarkably reduced when Akt was inhibited. These results reveal that Akt and HSF1 have a direct binding relationship, and that TMZ promotes the synergistic nuclear translocation of Akt and HSF1 by activating Akt.

Trimetazidine directly bound to Akt and activated Akt

As Akt was determined to be upstream of HSF1, we then asked whether TMZ directly binds to Akt. Schrodinger Suites 2018 was applied to examine the molecular docking of TMZ and Akt. YS-49, a known Akt agonist, was used as a positive control; as shown in Fig. 6a, YS-49 was located inside and completely wrapped by Akt. It combined with the Asp292, Trp80, and Tyr272 sites in Akt, forming two hydrogen bonds with Asp292 and Tyr272, and a pi-pi bond with Trp80, yielding a binding affinity of $-9.04 \mathrm{kcal} / \mathrm{mol}$. Similar to YS-49, TMZ was also located inside Akt and wrapped by it. Among the three core sites to which YS-49 and Akt bound, two residues bound with $\mathrm{TMZ}$; TMZ formed a hydrogen bond with 


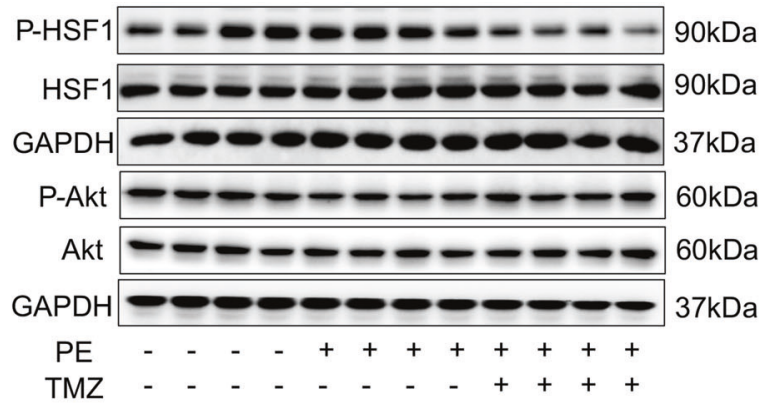

d

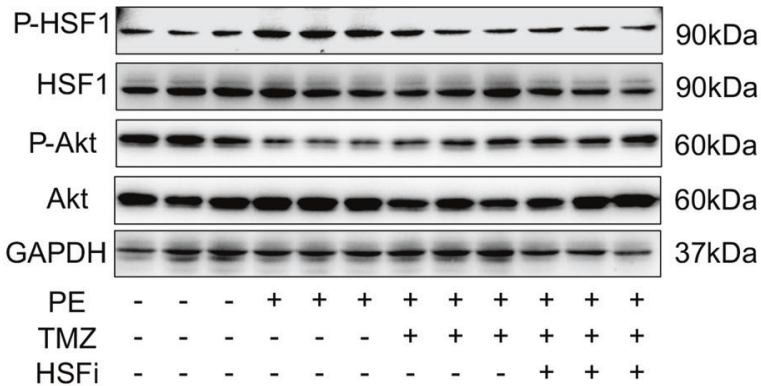

g

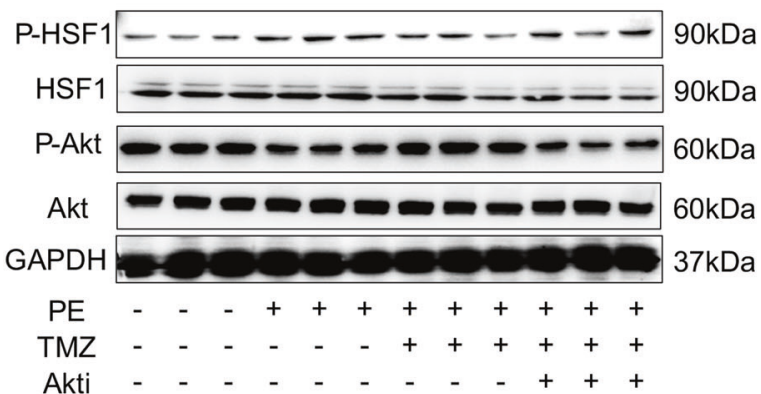

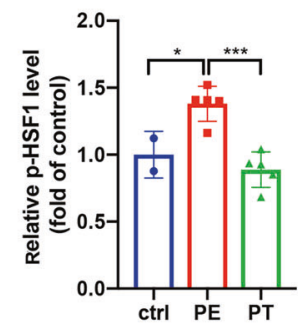

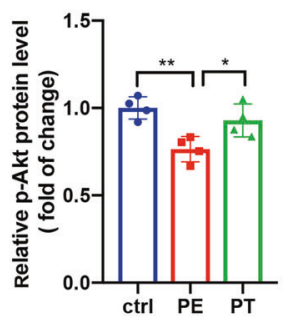

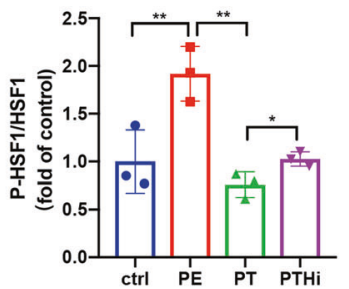

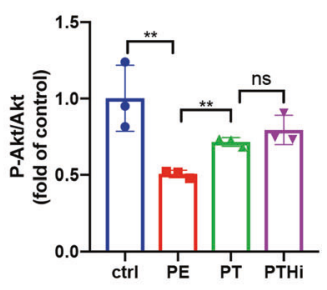

h

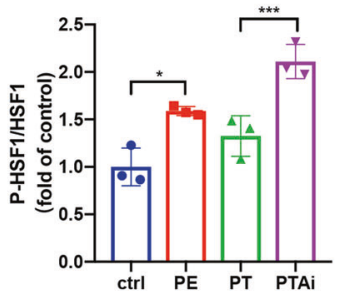

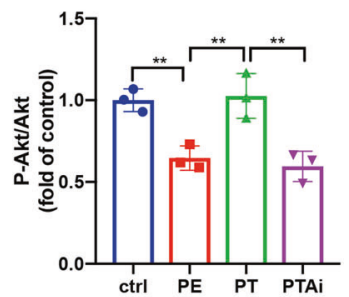

Fig. 4 TMZ triggered HSF1 via the activation of Akt. a Western blotting images of the phosphorylation of Akt and HSF1 in HUVECs exposed to PE with or without TMZ, GAPDH was used as a loading control. b Quantitative analysis of Western blotting images of p-HSF1 and HSF1 in (a). c Quantitative analysis of Western blotting images of p-Akt and Akt in (a). d Western blotting analysis of the phosphorylation of Akt and HSF1 in HUVECs exposed to PE and TMZ with or without HSF1 inhibitor (KRIBB11), GAPDH was used as a loading control. e Quantitative analysis of Western blotting images of p-HSF1 and HSF1 in (d). $\mathbf{f}$ Quantitative analysis of Western blotting images of p-Akt and Akt in (d). g Western blotting analysis of the phosphorylation of Akt and HSF1 in HUVECs exposed to PE and TMZ with or without Akt inhibitor (AZD5363), GAPDH was used as a loading control. $\mathbf{h}$ Quantitative analysis of Western blotting images of p-HSF1 and HSF1 in (g). i Quantitative analysis of Western blotting images of p-Akt and Akt in (g). Ctrl PBS group, PE Norepinephrine group, PT PE + TMZ group, cells treated with PE and then treated with TMZ, PTHi PE + TMZ + HSFi group, cells were treated with HSF1 inhibitor (KRIBB11), TMZ and PE, PTAi: PE + TMZ + Akti group, cells were treated with Akt inhibitor (AZD5363), TMZ and PE. Data were analyzed by two-way ANOVA analysis using GraphPad Prism 8 software $\left({ }^{*} P<0.5 ;{ }^{* *} P<0.01 ;{ }^{* *} P<0.01\right.$ vs. indicated group).

Asp292, and a pi-pi bond with Trp80, yielding a binding affinity of $-5.995 \mathrm{kcal} / \mathrm{mol}$. TMZ and YS-49 overlapped on two binding sites with relatively high binding affinities, illustrating that TMZ directly activates Akt. To validate whether Trp80 and Asp292 are critical amino acids, two mutational plasmids (Akt ${ }^{\mathrm{W} 80 \mathrm{~A}}$ and $A \mathrm{At}^{\mathrm{D} 292 \mathrm{~A}}$ ) were constructed. Fig. 6b, $c$ shows that neither Akt ${ }^{\text {W80A }}$ nor $A k t D^{292 A}$ responded to $T M Z$, unlike $A k t^{W T}$, suggesting that these two amino acids played a vital role in the activation of Akt by TMZ. Taken together, the above results show that TMZ directly activates Akt in endothelial cells via its binding with Trp80 and Asp292.

\section{DISCUSSION}

In this study, we demonstrate that TMZ binds to the Trp80 and Asp292 sites of Akt and subsequently activates Akt. It promotes the binding of Akt and HSF1, as well as the nuclear translocation of HSF1, by activating Akt. TMZ enhances HSF1-VEGF-A promoter region binding, thereby improving the angiogenesis dysfunction caused by pressure overload and promoting cardiac function. Our proposed model for the mechanism by which TMZ exerts its protective effect against cardiac angiogenesis is summarized in Fig. 7.

TMZ is widely used in the clinical treatment of cardiovascular diseases, including angina pectoris, ischemia-reperfusion, diabetic cardiomyopathy, and heart failure $[35,36]$. TMZ is reported to exert its protective effects through various mechanisms. It prevents diabetic cardiomyopathy by inhibiting NOX2/TrPC3induced oxidative stress [37], and protects against atherosclerosis by reducing reactive oxygen species production and regulating smooth muscle proliferation [38]. In addition, TMZ has also been implicated in the regulation of mitochondrial metabolism, 


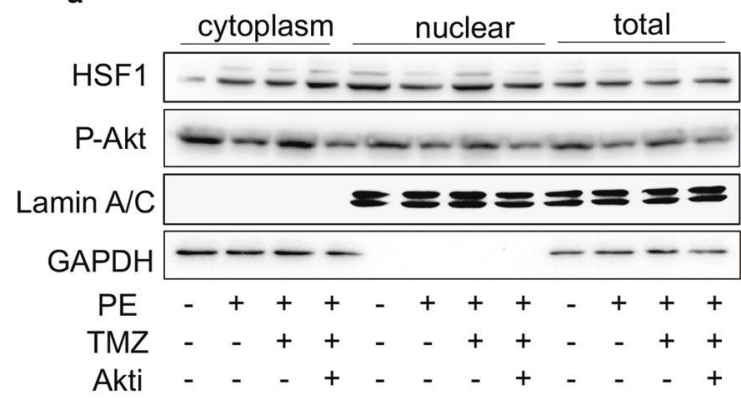

b

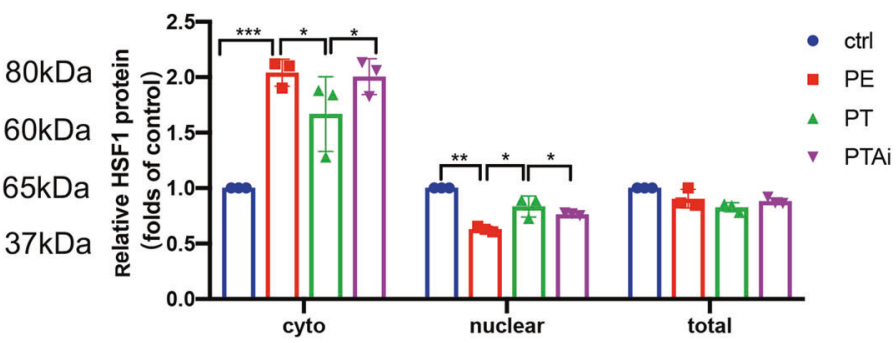

C
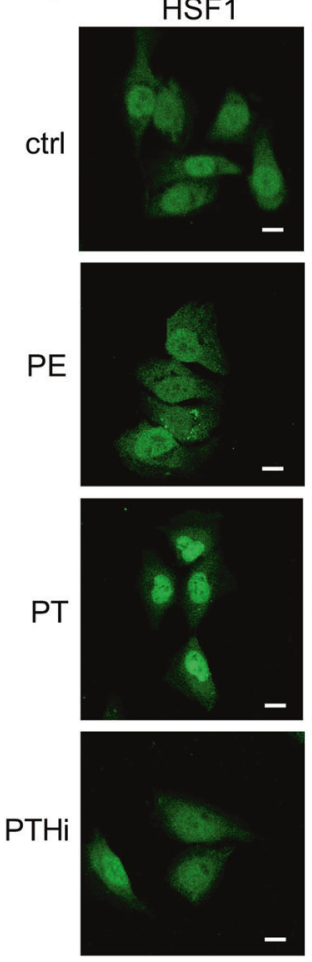

DAPI
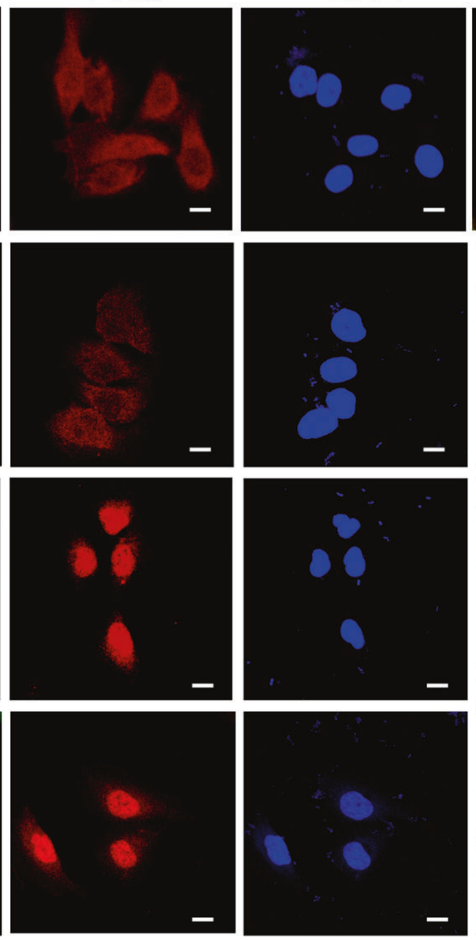
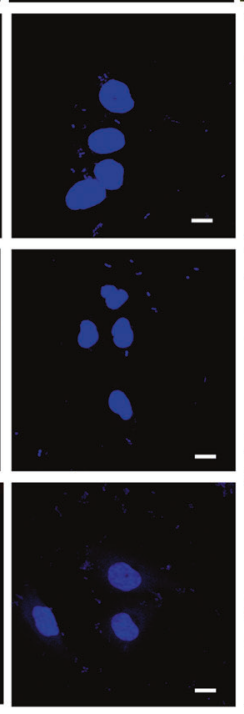

merge
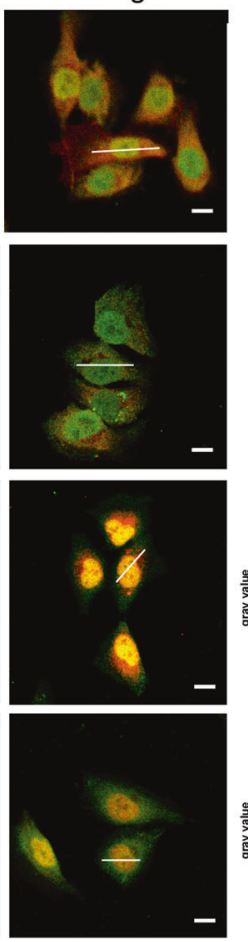

d

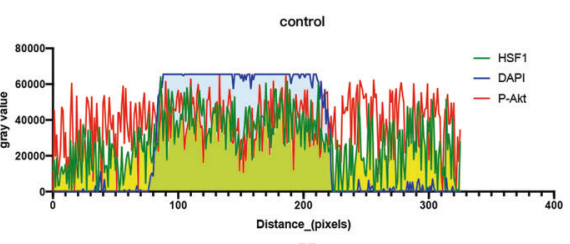

PE

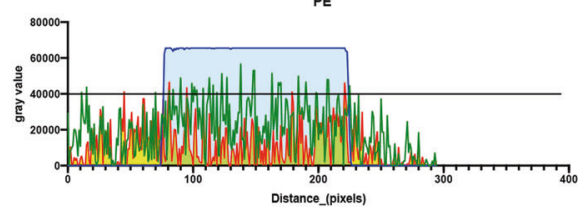

PT
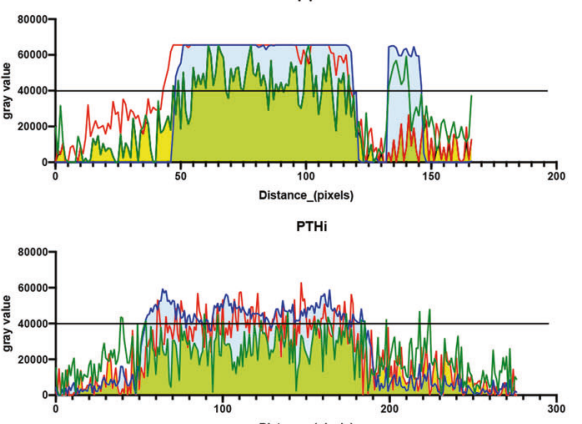

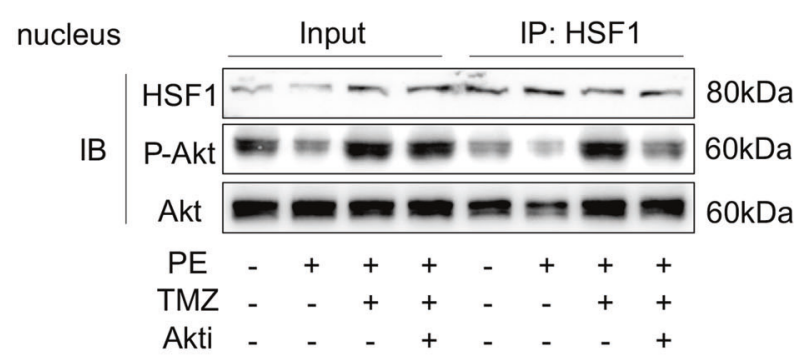

\section{f}

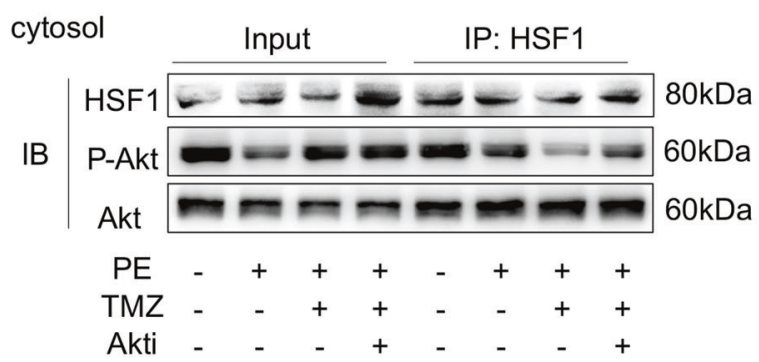

Fig. 5 TMZ promoted the nuclear translocation and binding of Akt and HSF1. a Western blotting analysis of the phosphorylation of Akt and HSF1 in the cytoplasm and nuclear of HUVECs exposed to PE and TMZ with or without Akt inhibitor (AZD5363), GAPDH was used as a total and cytoplasm loading control, Lamin A/C was used as a nuclear loading control. b Quantitative analysis of Western blotting images in (a). c Immunofluorescence staining with the p-Akt antibody (red) and HSF1 (green) of HUVECs. d Quantitative analysis of immunofluorescence intensity in indicated line. e, $\mathbf{f}$ Immunoprecipitation assays showing the interactions between p-Akt1 and HSF1 in HUVECs. Nuclear extracts and cytosol of HUVECs were obtained from indicated groups. Ctrl PBS group, PE Norepinephrine group, PT PE + TMZ group, cells treated with $\mathrm{PE}$ and then treated with TMZ, PTHi PE + TMZ + HSFi group, cells were treated with HSF1 inhibitor (KRIBB11), TMZ and PE, PTAi PE + TMZ + Akti group, cells were treated with Akt inhibitor (AZD5363), TMZ and PE. Data were analyzed by two-way ANOVA analysis using GraphPad Prism 8 software $\left({ }^{*} P<0.5 ;{ }^{* *} P<0.01 ;{ }^{* *} P<0.01\right.$ vs. indicated group). 

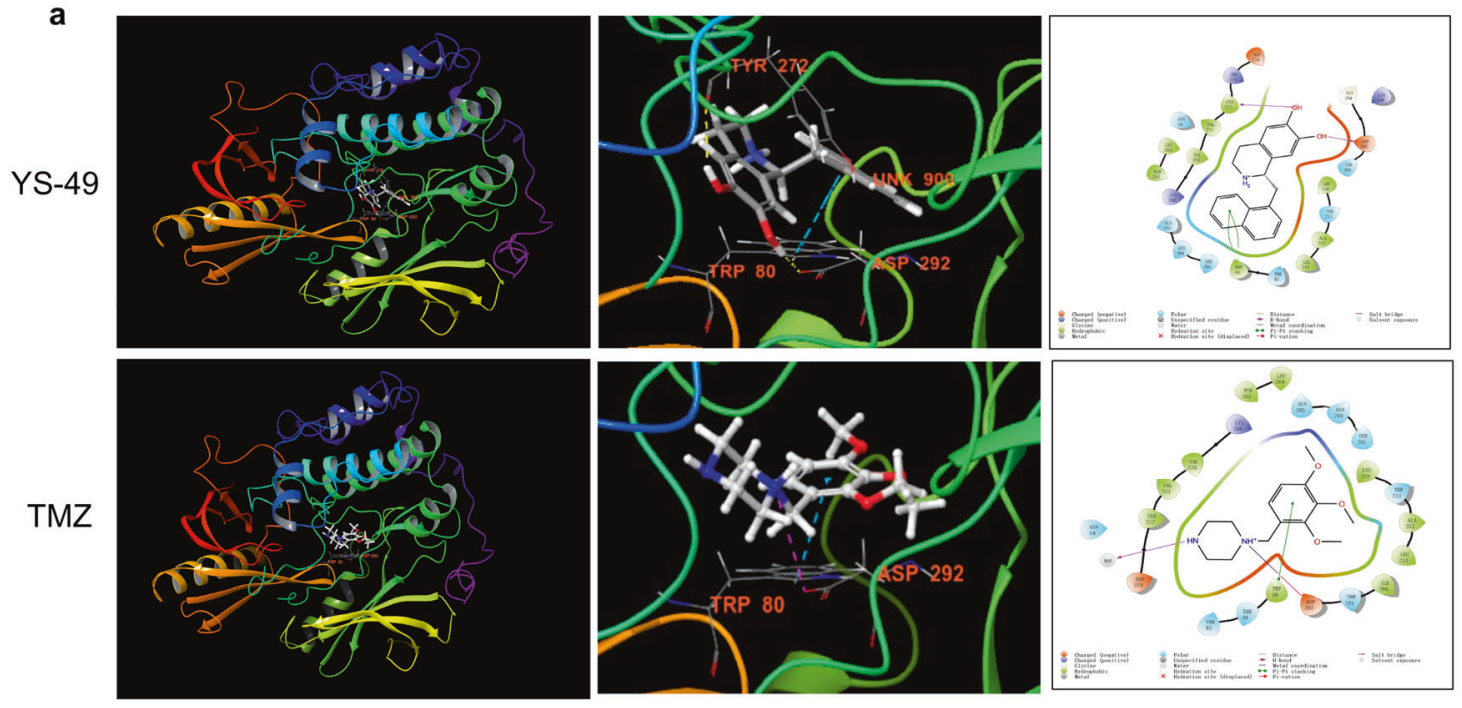

b

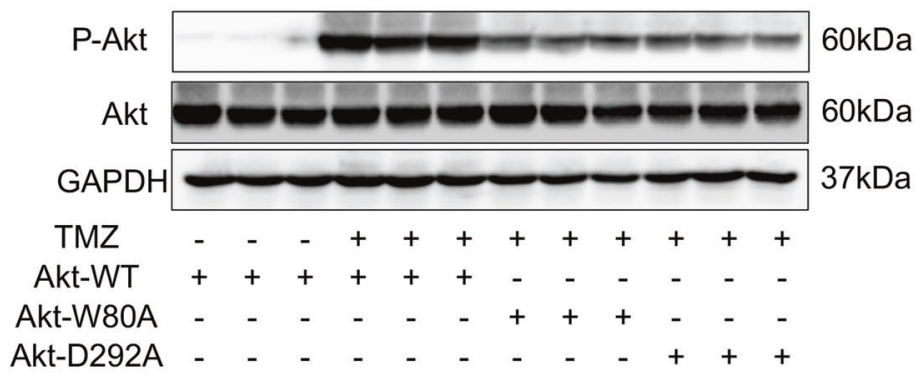

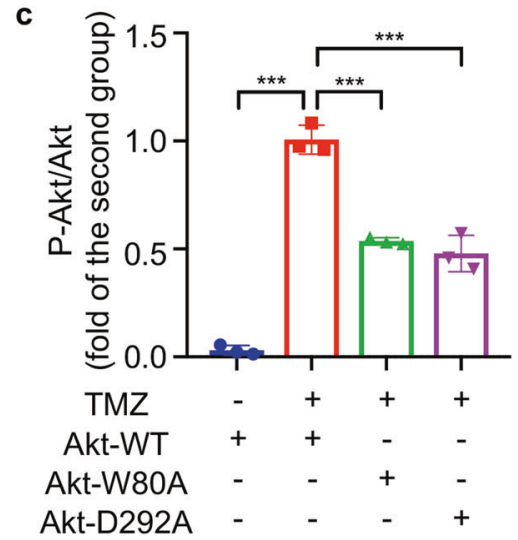

Fig. 6 TMZ bound to Akt in Trp80 and Asp292. a Upper, known agonist of Akt (YS-49) binds to Akt in Trp80, Asp292, and Tyr272. Lower, TMZ binds to Akt in Trp80 and Asp292. b Western blotting analysis of the phosphorylation of Akt in 293T cells transfected with indicated plasmids. GAPDH was used as a loading control. c Quantitative analysis of Western blotting images of (b). Data were analyzed by Student's $t$ test using GraphPad Prism 8 software (***P $<0.001$ vs. indicated group).

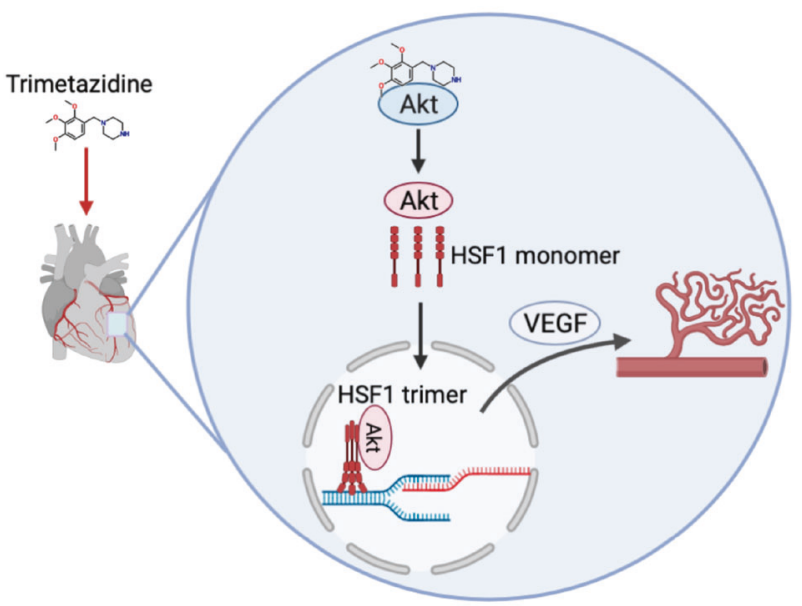

Fig. 7 Schematic overview of the proposed mechanism. TMZ activated Akt, and promoted the binding of Akt to HSF1, leading to the activation and nuclear translocation of HSF1. Activated HSF1 bound with the promoter region of VEGF-A, and eventually promoted its expression and angiogenesis. mitochondrial biosynthesis, and mitochondrial fission/fusion $[39,40]$. In contrast to previous studies, our study confirms that TMZ attenuates cardiac hypertrophy and heart failure by improving angiogenesis dysfunction. TMZ treatment increases blood vessel density and enhances VEGF expression, both in vivo and in vitro.

Akt is a serine/threonine kinase member of the AGC protein family and is widely involved in many cellular processes, including energy metabolism, angiogenesis, cell proliferation, and tumor growth. Thus, Akt is considered one of the most important signaling molecules in eukaryotic cells [41]. Trp80 and Asp292 are the two key amino acid residues that regulate Akt activity. Trp80 is the critical residue in the docking of MK-2206 with Akt [42]. When Trp80 is mutated to form alanine, Akt is resistant to its inhibitors [43]. Monocrotophos organophosphate pesticides inhibit Akt owing to the strong hydrogen bond between the Glu234 site of the monocrotophos and the Asp292 site of Akt [44]. As a wellknown metabolism modulating agent, TMZ also acts on Akt. For example, TMZ upregulates p-Akt in myocardial ischemia/reperfusion injury [13], and protects umbilical cord mesenchymal stem cells against hypoxia and serum deprivation-induced apoptosis by activating Akt [45]. Our previous study also revealed that TMZ activates Akt in a dose-dependent manner. However, none of 
these studies clarified the direct interaction between Akt and TMZ. Using the molecular docking software Schrodinger Suites 2018, we found that, similar to the working mode of YS-49 (an Akt agonist [46]), TMZ formed a hydrogen bond and a pi-pi bond with the Asp292 and Trp80 sites of Akt, respectively. Further experiments conducted in HUVECs provided solid evidence that both Asp292 and Trp80 are crucial for the activation of Akt.

As a typical transcription factor, the activity of HSF1 was determined by its abundance, protein modification, and subcellular distribution [47]. There were at least 12 phosphorylation sites in HSF1, of which Ser307 and Ser303 are well-established inhibitory phosphorylation sites. This implies that HSF1 activity decreases when phosphorylation levels of Ser307 and Ser303 increase [48]. Inactive HSF1 localizes in the cytosol and nucleus as a monomer. When HSF1 is activated, it changes its form from a monomer to a trimer and then translocates from the cytosol into the nucleus [47]. In the present study, the inhibitory phosphorylation level of HSF1 in HUVECs was significantly downregulated by TMZ treatment, accompanied by its enhanced nuclear accumulation, indicating that HSF1 was activated by TMZ. However, the activation of HSF1 was counterbalanced by the addition of Akt inhibitor, suggesting that HSF1 is not directly activated by TMZ, but is rather activated via Akt.

Several molecules are involved in the regulation of HSF1 activity. ERK inhibits HSF1 by directly phosphorylating HSF1 at Ser307 [49], while GSK3 $\beta$ exerts its inhibitory effect on HSF1 by phosphorylating Ser303 [50]. Sirt1 acts as an upstream molecule of HSF1 to reduce the acetylation level of HSF1 and activate it [51]. In the present study, enhanced HSF1 activity was accompanied by increased Akt activity. Inhibiting Akt restrained the activation of HSF1, whereas inhibiting HSF1 had no effects on the activity of Akt. These results suggest that Akt is critically involved in the regulation of HSF1 activity. Co-IP and immunofluorescence experiments further confirmed that Akt binds with HSF1. When Akt was activated by TMZ, it cooperated with HSF1 to aid its translocation to the nucleus, further exerting its regulatory effects.

HSF1 plays an essential role in autophagy, aging, development, metabolism, and immunity $[47,52]$. In the heart, HSF1 induces the apoptosis of senescent myocardial mitochondria by activating Omi/HtrA2 transcription [53], and acts as the main defender against palmitic acid-induced myocardial ferroptosis [54]. The latest research has confirmed that HSF1 promotes myocardial angiogenesis through the HIF-1a/VEGF pathway [33, 55]. Consistently, in our study, HSF1 activity increased with TMZ treatment under pressure overload, accompanied by an increase in myocardial blood vessel density, whereas the blood vessel density decreased with HSF1 inhibition.

Myocardial angiogenesis is regulated by a series of growthpromoting factors, including VEGF, angiopoietin-1 and -2 , fibroblast growth factors, transforming growth factors, and platelet-derived growth factors [7]. Among them, VEGF is the primary regulator of myocardial angiogenesis [56]. VEGF-deficient mice were more likely to transition from pathological myocardial hypertrophy to heart failure after TAC surgery [57]. In contrast, VEGF overexpression improved the contractility of failing hearts [58]. The present study reveals that, as a transcription factor, HSF1 binds with heat shock elements in the promoter region of VEGF-A and participates in the regulation of VEGF expression, providing direct evidence of the close relationship between HSF1 and angiogenesis.

Owing to technical limitations, this study has several shortcomings. First, instead of generating HSF1-KO mice, we intraperitoneally injected the mice with the HSF1 inhibitor, KRIBB11. KRIBB11 may have potential off-target effects. Second, we demonstrated that TMZ formed hydrogen and pi-pi bonds with Akt using molecular docking analysis. However, we did not simulate the dynamic molecular conformation of Akt when combined with TMZ. Future studies are needed to elucidate the molecular conformational changes that occur when TMZ binds with Akt.

Our proposed model, based on the above data, is shown in Fig. 7. After TMZ enters the endothelial cells, it activates Akt by binding with its Asp292 and Trp80 residues. Then, the activated Akt activates and promotes the nuclear translocation of HSF1. By combining with the promoter region of VEGF-A, HSF1 stimulates the expression of VEGF-A, and ultimately attenuates angiogenesis dysfunction and promotes cardiac function under pressure overload. In summary, our study demonstrates that HSF1 mediates the protective effects of TMZ in promoting angiogenesis, and provides a new theoretical basis for the cardio-protective functions of TMZ.

\section{ACKNOWLEDGEMENTS}

We thank Dr. Zheng Wen and Dr. Ying-hui Wang (Division of Cardiology, Department of Internal Medicine, Tongji Hospital, Tongji Medical College, Huazhong University of Science and Technology) who kindly helped the animal experiments. This work was supported by National Natural Science Foundation of China (No. 82070316, 81570261, 82000383), and Cardiovascular Health Alliance of China-Advanced Fund (2019-CCA-ACCESS-059).

\section{AUTHOR CONTRIBUTIONS}

HYS designed and performed all the experiments and wrote the paper draft; YZP and LS helped the molecular docking of trimetazidine/YS-49 with Akt and the paper writing; WJH helped the animal experiments and results discussion; MZ conducted the tube formation assay. NZ provided financial supports, designed the study, and finished final writing of the paper; DWW provided the laboratory where experiments being conducted.

\section{ADDITIONAL INFORMATION}

Supplementary information The online version contains supplementary material available at https://doi.org/10.1038/s41401-022-00877-8.

Competing interests: The authors declare no competing interests.

\section{REFERENCES}

1. Chow CK, Teo KK, Rangarajan S, Islam S, Gupta R, Avezum A, et al. Prevalence, awareness, treatment, and control of hypertension in rural and urban communities in high-, middle-, and low-income countries. JAMA. 2013;310:959-68. https://doi.org/10.1001/jama.2013.184182.

2. Kearney PM, Whelton M, Reynolds K, Muntner P, Whelton PK, He. J. Global burden of hypertension: analysis of worldwide data. Lancet. 2005;365:217-23. https://doi. org/10.1016/S0140-6736(05)17741-1.

3. Muntner P, Hardy ST, Fine LJ, Jaeger BC, Wozniak G, Levitan EB, et al. Trends in blood pressure control among US adults with hypertension, 1999-2000 to 2017 2018. JAMA. 2020;324:1190-200. https://doi.org/10.1001/jama.2020.14545.

4. Ziaeian B, Fonarow GC. Epidemiology and aetiology of heart failure. Nat Rev Cardiol. 2016;13:368-78. https://doi.org/10.1038/nrcardio.2016.25.

5. Boudina S, Abel ED. Diabetic cardiomyopathy revisited. Circulation. 2007;115:3213-23. https://doi.org/10.1161/CIRCULATIONAHA.106.679597.

6. Orso F, Fabbri G, Pietro Maggioni A. Epidemiology of heart failure. Handb Exp Pharmacol. 2017;243:15-33. https://doi.org/10.1007/164 201674.

7. Oka T, Akazawa H, Naito AT, Komuro. I. Angiogenesis and cardiac hypertrophy: maintenance of cardiac function and causative roles in heart failure. Circ Res. 2014;114:565-71. https://doi.org/10.1161/CIRCRESAHA.114.300507.

8. Shigeta T, Aoyama M, Bando YK, Monji A, Mitsui T, Takatsu M, et al. Dipeptidyl peptidase-4 modulates left ventricular dysfunction in chronic heart failure via angiogenesis-dependent and -independent actions. Circulation. 2012;126:1838-51. https://doi.org/10.1161/CIRCULATIONAHA.112.096479.

9. Milinković I, Rosano G, Lopatin Y, Seferović PM. The role of ivabradine and trimetazidine in the New ESC HF Guidelines. Card Fail Rev. 2016;2:123-9. https://doi. org/10.15420/cfr.2016:13:1.

10. Li H, Ma Z, Zhai Y, Lv C, Yuan P, Zhu F, et al. Trimetazidine ameliorates myocardial metabolic remodeling in isoproterenol-induced rats through regulating ketone body metabolism via activating AMPK and PPAR a. Front Pharmacol. 2020;11:1255. https://doi.org/10.3389/fphar.2020.01255. 
11. Chu Y-S, Li D-X, Zhang M, Jiang. T-M. Trimetazidine hydrochloride as a new treatment for patients with peripheral vascular disease-an exploratory trial. Eur Rev Med Pharmacol Sci. 2016;20:188-93.

12. Lian X, He W, Zhan H, Chen J, Tan N, He P, et al. The effect of trimetazidine on preventing contrast-induced nephropathy after cardiac catheterization. Int Urol Nephrol. 2019;51:2267-72. https://doi.org/10.1007/s11255-019-02308-w.

13. Wu S, Chang G, Gao L, Jiang D, Wang L, Li G, et al. Trimetazidine protects against myocardial ischemia/reperfusion injury by inhibiting excessive autophagy. J Mol Med. 2018;96:791-806. https://doi.org/10.1007/s00109-018-1664-3.

14. Rosano GM, Vitale. C. Metabolic modulation of cardiac metabolism in heart failure. Card Fail Rev. 2018;4:99-103. https://doi.org/10.15420/cfr.2018.18.2.

15. Kantor PF, Lucien A, Kozak R, Lopaschuk. GD. The antianginal drug trimetazidine shifts cardiac energy metabolism from fatty acid oxidation to glucose oxidation by inhibiting mitochondrial long-chain 3-ketoacyl coenzyme A thiolase. Circ Res 2000;86:580-8. https://doi.org/10.1161/01.res.86.5.580.

16. Lopaschuk GD, Barr R, Thomas PD, Dyck. JRB. Beneficial effects of trimetazidine in ex vivo working ischemic hearts are due to a stimulation of glucose oxidation secondary to inhibition of long-chain 3-ketoacyl coenzyme a thiolase. Circ Res. 2003;93:e33-7. https://doi.org/10.1161/01.RES.0000086964.07404.A5.

17. Liu Y-C, Li L, Su Q, Liu T, Tang. Z. Trimetazidine pretreatment inhibits myocardial apoptosis and improves cardiac function in a Swine model of coronary microembolization. Cardiology. 2015;130:130-6. https://doi.org/10.1159/000369246.

18. Zheng W, Liu C. The cystathionine gamma-lyase/hydrogen sulfide pathway mediates the trimetazidine-induced protection of H9c2 cells against hypoxia/ reoxygenation-induced apoptosis and oxidative stress. Anatol J Cardiol. 2019;22:102-11. https://doi.org/10.14744/AnatolJCardiol.2019.83648.

19. Liu X, Gai Y, Liu F, Gao W, Zhang Y, Xu M, et al. Trimetazidine inhibits pressure overload-induced cardiac fibrosis through NADPH oxidase-ROS-CTGF pathway. Cardiovasc Res. 2010;88:150-8. https://doi.org/10.1093/cvr/cvq181.

20. Zhao Y, Li S, Quan E, Zhang H, Wu Y, Luo Y, et al. Trimetazidine inhibits cardiac fibrosis by reducing reactive oxygen species and downregulating connective tissue growth factor in streptozotocin-induced diabetic rats. Exp Ther Med. 2019;18:1477-85. https://doi.org/10.3892/etm.2019.7705

21. Chen J, Wang B, Lai J, Braunstein Z, He M, Ruan G, et al. Trimetazidine attenuates cardiac dysfunction in endotoxemia and sepsis by promoting neutrophil migration. Front Immunol. 2018;9:2015. https://doi.org/10.3389/fimmu.2018.02015.

22. Zhou X, Li C, Xu W, Chen J. Trimetazidine protects against smoking-induced left ventricular remodeling via attenuating oxidative stress, apoptosis, and inflammation. PLoS One. 2012;7:e40424. https://doi.org/10.1371/journal.pone.0040424.

23. Ferrari R, Ford I, Fox K, Challeton JP, Correges A, Tendera M, et al. Efficacy and safety of trimetazidine after percutaneous coronary intervention (ATPCI): a randomised, double-blind, placebo-controlled trial. Lancet. 2020;396:830-8. https:// doi.org/10.1016/S0140-6736(20)31790-6.

24. Lai KK, Gang DL, Zawacki JK, Cooley TP. Fulminant hepatic failure associated with 2',3'-dideoxyinosine (ddl). Ann Intern Med. 1991;115:283-4. https://doi.org/ 10.7326/0003-4819-115-4-283.

25. Sastry GM, Adzhigirey M, Day T, Annabhimoju R, Sherman W. Protein and ligand preparation: parameters, protocols, and influence on virtual screening enrichments. J Comput Aided Mol Des. 2013;27:221-34. https://doi.org/10.1007/ s10822-013-9644-8.

26. Lu S, Strand KA, Mutryn MF, Tucker RM, Jolly AJ, Furgeson SB, et al. PTEN (Phosphatase and Tensin Homolog) protects against Ang II (Angiotensin II)-induced pathological vascular fibrosis and remodeling-brief report. Arterioscler Thromb Vasc Biol. 2020;40:394-403. https://doi.org/10.1161/ATVBAHA.119.313757.

27. Zheng $\mathrm{H}$, Tie $\mathrm{Y}$, Fang $\mathrm{Z}, \mathrm{Wu} X, \mathrm{Yi}$ T, Huang $\mathrm{S}$, et al. Jumonji domain-containing 6 (JMJD6) identified as a potential therapeutic target in ovarian cancer. Signal Transduct Target Ther. 2019;4:24. https://doi.org/10.1038/s41392-019-0055-8.

28. Sun J, Shen H, Shao L, Teng X, Chen $Y$, Liu X, et al. HIF-1a overexpression in mesenchymal stem cell-derived exosomes mediates cardioprotection in myocardial infarction by enhanced angiogenesis. Stem Cell Res Ther. 2020;11:373. https://doi.org/10.1186/s13287-020-01881-7.

29. Chen $\mathrm{H}$, Long $\mathrm{H}$, Cui $X$, Zhou J, Xu M, Yuan G. Exploring the formation and recognition of an important G-quadruplex in a HIF1a promoter and its transcriptional inhibition by a benzo[c]phenanthridine derivative. J Am Chem Soc. 2014;136:2583-91. https://doi.org/10.1021/ja412128w.

30. Rodriguez LG, Wu X, Guan J-L. Wound-healing assay. Methods Mol Biol. 2005;294:23-9. https://doi.org/10.1385/1-59259-860-9:023.

31. Behnammanesh G, Durante ZE, Peyton KJ, Martinez-Lemus LA, Brown SM, Bender SB, et al. Canagliflozin inhibits human endothelial cell proliferation and tube formation. Front Pharmacol. 2019;10:362. https://doi.org/10.3389/fphar.2019.00362.

32. Wertz SL, Desimine VL, Maning J, McCrink KA, Lymperopoulos A. Co-IP assays for measuring GPCR-arrestin interactions. Methods Cell Biol. 2019;149:205-13. https://doi.org/10.1016/bs.mcb.2018.09.003.

33. Tian X, Zhou N, Yuan J, Lu L, Zhang Q, Wei M, et al. Heat shock transcription factor 1 regulates exercise-induced myocardial angiogenesis after pressure overload via
HIF-1a/VEGF pathway. J Cell Mol Med. 2020;24:2178-88. https://doi.org/10.1111/ jcmm.14872.

34. Zou Y, Li J, Ma H, Jiang H, Yuan J, Gong H, et al. Heat shock transcription factor 1 protects heart after pressure overload through promoting myocardial angiogenesis in male mice. J Mol Cell Cardiol. 2011;51:821-9. https://doi.org/10.1016/j. yjmcc.2011.07.030.

35. McCarthy CP, Mullins KV, Kerins DM. The role of trimetazidine in cardiovascular disease: beyond an anti-anginal agent. Eur Hear J Cardiovasc Pharmacother. 2016;2:266-72. https://doi.org/10.1093/ehjcvp/pvv051.

36. Marzilli M, Vinereanu D, Lopaschuk G, Chen Y, Dalal JJ, Danchin N, et al. Trimetazidine in cardiovascular medicine. Int J Cardiol. 2019;293:39-44. https://doi.org/ 10.1016/j.ijcard.2019.05.063.

37. Tang S-G, Liu X-Y, Wang S-P, Wang H-H, Jovanovic A, Tan W. Trimetazidine prevents diabetic cardiomyopathy by inhibiting Nox2/TRPC3-induced oxidative stress. J Pharmacol Sci. 2019;139:311-8. https://doi.org/10.1016/j.jphs.2019.01.016.

38. Zheng S, Du Y, Peng Q, Fan X, Li J, Chen M. Trimetazidine protects against atherosclerosis by changing energy charge and oxidative stress. Med Sci Monit Int Med J Exp Clin Res. 2018;24:8459-68. https://doi.org/10.12659/MSM.911317.

39. Shi W, Shangguan W, Zhang Y, Li C, Li G. Effects of trimetazidine on mitochondrial respiratory function, biosynthesis, and fission/fusion in rats with acute myocardial ischemia. Anatol J Cardiol. 2017;18:175-81. https://doi.org/10.14744/ AnatolJCardiol.2017.7771.

40. Kuzmicic J, Parra V, Verdejo HE, López-Crisosto C, Chiong M, García L, et al. Trimetazidine prevents palmitate-induced mitochondrial fission and dysfunction in cultured cardiomyocytes. Biochem Pharmacol. 2014;91:323-36. https://doi.org/ 10.1016/j.bcp.2014.07.022.

41. Manning BD, Toker A. AKT/PKB signaling: navigating the network. Cell. 2017;169:381-405. https://doi.org/10.1016/j.cell.2017.04.001.

42. Rehan M, Beg MA, Parveen S, Damanhouri GA, Zaher GF. Computational insights into the inhibitory mechanism of human AKT1 by an orally active inhibitor, MK2206. PLoS One. 2014;9:e109705. https://doi.org/10.1371/journal.pone.0109705.

43. Green CJ, Göransson O, Kular GS, Leslie NR, Gray A, Alessi DR, et al. Use of Akt inhibitor and a drug-resistant mutant validates a critical role for protein kinase B/Akt in the insulin-dependent regulation of glucose and system A amino acid uptake. J Biol Chem. 2008;283:27653-67. https://doi.org/10.1074/jbc.M802623200.

44. Kashyap MP, Singh AK, Kumar V, Yadav DK, Khan F, Jahan S, et al. Pkb/Akt1 mediates Wnt/GSK3 $\beta / \beta$-catenin signaling-induced apoptosis in human cord blood stem cells exposed to organophosphate pesticide monocrotophos. Stem Cells Dev. 2013;22:224-38. https://doi.org/10.1089/scd.2012.0220.

45. Gong X, Fan G, Wang W, Wang G. Trimetazidine protects umbilical cord mesenchymal stem cells against hypoxia and serum deprivation induced apoptosis by activation of Akt. Cell Physiol Biochem. 2014;34:2245-55. https://doi.org/ 10.1159/000369667.

46. Hsu Y-H, Chang C-C, Yang N-J, Lee Y-H, Juan S-H. RhoA-mediated inhibition of vascular endothelial cell mobility: positive feedback through reduced cytosolic p21 and p27. J Cell Physiol. 2014:229:1455-65. https://doi.org/10.1002/jcp.24583.

47. Li J, Labbadia J, Morimoto RI. Rethinking HSF1 in stress, development, and organismal health. Trends Cell Biol. 2017;27:895-905. https://doi.org/10.1016/j. tcb.2017.08.002.

48. Xu Y-M, Huang D-Y, Chiu J-F, Lau ATY. Post-translational modification of human heat shock factors and their functions: a recent update by proteomic approach. J Proteome Res. 2012;11:2625-34. https://doi.org/10.1021/pr201151a.

49. Wang X, Grammatikakis N, Siganou A, Stevenson MA, Calderwood SK. Interactions between extracellular signal-regulated protein kinase 1, 14-3-3epsilon, and heat shock factor 1 during stress. J Biol Chem. 2004;279:49460-9. https://doi.org/ 10.1074/jbc.M406059200.

50. Xavier IJ, Mercier PA, McLoughlin CM, Ali A, Woodgett JR, Ovsenek N. Glycogen synthase kinase 3 beta negatively regulates both DNA-binding and transcriptional activities of heat shock factor 1. J Biol Chem. 2000;275:29147-52. https://doi.org/ 10.1074/jbc.M002169200.

51. Westerheide SD, Anckar J, Stevens SMJ, Sistonen L, Morimoto RI. Stress-inducible regulation of heat shock factor 1 by the deacetylase SIRT1. Science. 2009;323:1063-6. https://doi.org/10.1126/science.1165946.

52. Barna J, Csermely P, Vellai T. Roles of heat shock factor 1 beyond the heat shock response. Cell Mol Life Sci. 2018;75:2897-916. https://doi.org/10.1007/s00018018-2836-6.

53. Liu D, Wu L, Wu Y, Wei X, Wang W, Zhang S, et al. Heat shock factor 1-mediated transcription activation of $\mathrm{Omi} / \mathrm{HtrA2}$ induces myocardial mitochondrial apoptosis in the aging heart. Aging. 2019;11:8982-97. https://doi.org/10.18632/ aging.102361.

54. Wang N, Ma H, Li J, Meng C, Zou J, Wang H, et al. HSF1 functions as a key defender against palmitic acid-induced ferroptosis in cardiomyocytes. J Mol Cell Cardiol. 2020;150:65-76. https://doi.org/10.1016/j.yjmcc.2020.10.010.

55. Wang S, Wu J, You J, Shi H, Xue X, Huang J, et al. HSF1 deficiency accelerates the transition from pressure overload-induced cardiac hypertrophy to heart failure 
through endothelial miR-195a-3p-mediated impairment of cardiac angiogenesis. J Mol Cell Cardiol. 2018;118:193-207. https://doi.org/10.1016/j.yjmcc.2018.03.017.

56. Matsumoto K, Ema M. Roles of VEGF-A signalling in development, regeneration, and tumours. J Biochem. 2014;156:1-10. https://doi.org/10.1093/jb/mvu031.

57. Izumiya Y, Shiojima I, Sato K, Sawyer DB, Colucci WS, Walsh K. Vascular endothelial growth factor blockade promotes the transition from compensatory cardiac hypertrophy to failure in response to pressure overload. Hypertension. 2006;47:887-93. https://doi.org/10.1161/01.HYP.0000215207.54689.31.

58. Friehs I, Barillas R, Vasilyev NV, Roy N, McGowan FX, del Nido PJ. Vascular endothelial growth factor prevents apoptosis and preserves contractile function in hypertrophied infant heart. Circulation. 2006;114:1290-5. https://doi.org/ 10.1161/CIRCULATIONAHA.105.001289.
Open Access This article is licensed under a Creative Commons Attribution 4.0 International License, which permits use, sharing, adaptation, distribution and reproduction in any medium or format, as long as you give appropriate credit to the original author(s) and the source, provide a link to the Creative Commons license, and indicate if changes were made. The images or other third party material in this article are included in the article's Creative Commons license, unless indicated otherwise in a credit line to the material. If material is not included in the article's Creative Commons license and your intended use is not permitted by statutory regulation or exceeds the permitted use, you will need to obtain permission directly from the copyright holder. To view a copy of this license, visit http://creativecommons. org/licenses/by/4.0/.

(c) The Author(s) 2022 